\title{
An analysis of pulse wave signals during visual display terminal operations
}

\author{
Yang Lu, Qi Wang, Song Zhang, Lin Yang*, Liyan Liang and Jingxuan Cui \\ College of Life Science and Bio-engineering, Beijing University of Technology, Beijing, 100124, \\ China
}

\begin{abstract}
In this paper, participants operated visual display terminals (VDT); the authors analyzed their pulse wave signals to develop objective indices for diagnosing and assessing VDT-induced. The experiment used 30 healthy undergraduates test subjects; each subject operated static PC for 4 hours. The pulse waves of local blood volumes in the test subjects' finger-tips were measured every 30 minutes for 2 minutes. After the experiment, the pulse wave parameters were analyzed. All test subjects exhibited signs of visual fatigue according to the fatigue scale results. There were significant differences of heart rate (HR), which clearly declined before and after the experiment $(\mathrm{P}<0.01)$. The indexes RMSSD, SDNN, P3, P4, P5, P6, and TP had significant differences $(\mathrm{P}<0.01)$ and all rose. The positions of the main and dicrotic waves advanced $(\mathrm{P}<0.01)$. The experiment parameters prompted significant changes; pulse wave forms changed during VDT work. Based on these results, pulse wave analysis holds promise as an effective, noninvasive technique for measuring VDT fatigue.
\end{abstract}

Keywords: Pulse wave, fatigue, visual display terminal (VDT), pulse wave characteristic values

\section{Introduction}

Video display terminals (VDT) are widely used in many fields [1]. They can lead to a variety of problems, such as physical discomfort (dry eyes or rising heart rates) [2], psychological discomfort (anxiety or frustration), and financial losses cause by increased error rates [3-5]. Methods such as analyzing EEG, eye signals, or ECG, could be used to evaluate user fatigue, but these methods are not effective for daily testing $[6,7]$.

The pulse wave contains abundant physiological information; information obtained from this method is respected in domestic and foreign academic circles [8]. It is a noninvasive detection method, and pulse parameters reflect changes in cardiovascular system; therefore, pulse wave detection holds promise as an efficient method detect VDT operation fatigue. Meanwhile, HRV is recognized as a reliable indicator for measuring stress levels $[9,10]$. However, it is inconvenient to measure HRV on a daily basis. Pulse rate variability (PRV) can be calculated by the adjacent peaks of pulse wave intervals; it also can be reflected the autonomic nervous system regulating process $[11,12]$. The pulse frequency variability and other frequency domain parameters must be detected $[13,14]$. Therefore, the

\footnotetext{
* Address for correspondence: Lin Yang, College of Life Science and Bio-engineering, Beijing University of Technology, No. 100 Pingleyuan, Chaoyang District, Beijing, China. Tel.: +8667392010; Fax: +8667392010; E-mail: yanglin@bjut.edu.cn.
} 
authors developed an experiment using pulse wave acquisition to detect VDT-induced fatigue.

\section{Materials and methods}

\subsection{Subjects}

All test subjects were healthy; they did not possess systemic diseases (such as high blood pressure, anemia, diabetes, or cardiac insufficiency), mental illness, early psychological disease, eye organ diseases, or strabismus refractive error diseases (such as glaucoma). The experiment used 20 male, and 10 female test subjects. All subjects were undergraduate or graduate students.

\subsection{Procedures}

The study was a randomized, double-blind trial. Subjects and operators did not know the study's objectives. Before the experiment, the operators were required to be familiar with the experiment's instruments and operation method. Subjects answered a questionnaire about their game process skills, their previous day's rest, and their current wakefulness. They also completed a fatigue scale and visual fatigue scale analysis. During the experiment, subjects operated the computer games Monte Zama Treasure for 4 hours with closed communication equipment. The subjects were in a relatively closed space. Every 30minutes for 2 minutes at a time, subjects' finger-tip volume pulse wave data was collected. After the test, the subjects completed a fatigue scale analysis, visual fatigue scale analysis and waveform collection.

The laboratory was at room temperature $(23 \pm 2.1)$, under USES white fluorescent lamp lighting, maintaining uniform brightness. The testing site was kept relatively quiet and maintained good ventilation.

The experiment used The ADInstruments PowerLab four-channel physiological signal acquisition system for data acquisition. Using with signal acquisition system Chart for Windows, finger-tip volume pulse wave data was recorded and displayed.

Experiments used a 14-point fatigue scale. It is composed of 14 items. 0 indicated the lowest fatigue level and 14 indicated the highest fatigue level.

The fatigue questionnaire was developed by Rechichi. Under this model, a fatigue score of 10 indicated symptoms such as headache, and stinging eyes.

\subsection{Experimental analysis parameters}

Pulse wave detection and analysis software was developed in a laboratory to collect finger-tip volume pulse wave, pulse frequency variation parameters such as RMSSD and SDNN, the times of main waves and replay wave valleys, the height of replay wave valleys, the total power, and the power of each harmonic. SPSS data analysis software was used to get the experiment's results.

The pulse heart rate refers to the pulse number in a pulse beat. It can be collected by determining the adjacent pulse wave crest spacing. This experiment used both time-domain pulse rate variability indexes: SDNN and RMSSD.

$$
S D N N=\sqrt{\frac{\sum_{i=0}^{N}(R R i-\text { meanRR })^{2}}{N}}
$$




$$
R M S S D=\sqrt{\frac{\sum_{\mathrm{i}=1}^{N-1}\left(R R_{i+1}-R R_{i}\right)^{2}}{N-1}}
$$

$\mathrm{N}$ is the total number of heart beats. $\mathrm{RRi}$ represents the sphygmicinterval. MeanRR is the sphygmicinterval average.

The experiment completed a large number of uniform pulse wave quantization. Each pulse waveform signal had 100 data points; the wave amplitude had 100 discrete quantitative values, forming the waveform quantitative plane, 100, 100.

The main wave peak of the pulse wave and the point of the dicrotic wave trough position are both crucial features of a pulse waveform. As shown in Figure 1, this study presents the main wave in time $\mathrm{T} 1$, the dicrotic wave trough appears in time T2, and the trough height is D2. These parameters were normalized after calculation.

The power spectrum of the pulse wave was analyzed by FFT. The main frequency of pulse waves concentrated on $0-15 \mathrm{~Hz}$. Therefore, the experiment only analyzed that range in the power spectrum.

As shown in Figure 2, there are peaks in the pulse wave power spectrum. The first wave frequency reflects the human pulse frequency, and the second wave frequency is 2 times of the first wave frequency, and so on; all wave frequencies are integer multiples of the first wave frequency. Therefore, these peaks are the harmonic of pulse frequencies. The six harmonics are the first harmonic to the sixth harmonic; their amplitudes are respectively P1, P2, P3, P4, P5, P6, and possessed an average power TP of $0-15 \mathrm{~Hz}$.

\subsection{Statistical analysis}

By using the SPSS19.0 statistical software, the measured values were expressed as mean \pm standard, and the standard slip (coefficient of variation) was calculated using the paired t-test statistical methods.

\section{Results}

All 30 subjects completed the fatigue scale before and after the experiment. Subjects' subjective

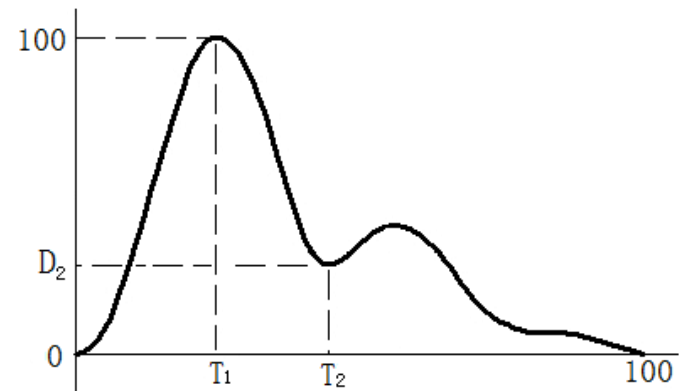

Fig. 1. The position of the main wave, and the position and height of the dicrotic wave trough.

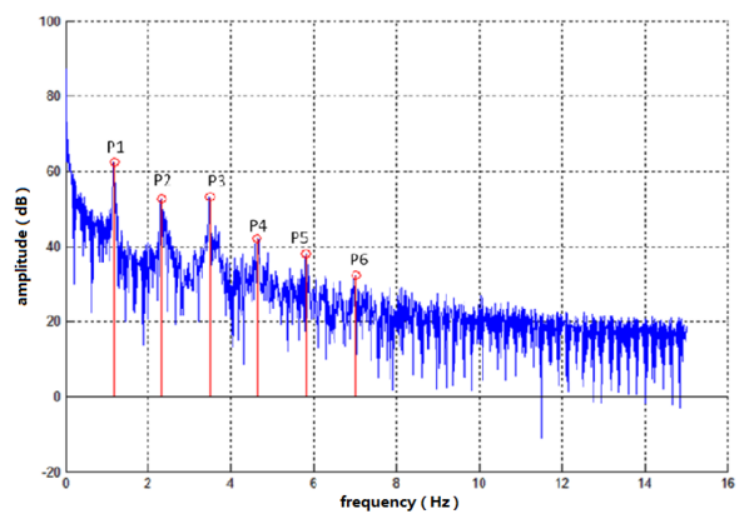

Fig. 2. Pulse wave power spectrum. 
Table 1

The mean value of $\mathrm{K}^{\prime}, \mathrm{T}_{1}, \mathrm{~T}_{2}, \mathrm{D}_{2}, \mathrm{HR}$, RMSSD, and SDNN

\begin{tabular}{|c|c|c|c|c|c|c|c|}
\hline $\begin{array}{l}\text { Time/ } \\
\text { Parameters }\end{array}$ & $\mathrm{K}^{\prime}$ & $\mathrm{T}_{1}$ & $\mathrm{~T}_{2}$ & $\mathrm{D}_{2}$ & HR & RMSSD & SDNN \\
\hline 0 & $0.546 \pm 0.009$ & $0.21 \pm 0.008$ & $0.43 \pm 0.010$ & $70.5 \pm 2.87$ & $79.5 \pm 7.9$ & $31.2 \pm 10.9$ & $28.4 \pm 7.0$ \\
\hline $0.5 \mathrm{~h}$ & $0.532 \pm 0.020$ & $0.18 \pm 0.009^{*}$ & $0.42 \pm 0.017$ & $69.6 \pm 2.73$ & $74.2 \pm 6.0^{* *}$ & $36.7 \pm 11.0 * *$ & $31.9 \pm 10.1 * *$ \\
\hline $1 \mathrm{~h}$ & $0.540 \pm 0.011$ & $0.17 \pm 0.009 * *$ & $0.39 \pm 0.016^{*}$ & $71.7 \pm 3.01$ & $73.0 \pm 6.9 * *$ & $38.8 \pm 12.4^{* *}$ & $33.3 \pm 8.5^{* *}$ \\
\hline $1.5 \mathrm{~h}$ & $0.557 \pm 0.009$ & $0.18 \pm 0.010^{* *}$ & $0.39 \pm 0.016^{*}$ & $76.0 \pm 2.45^{*}$ & $71.6 \pm 6.5 * *$ & $40.4 \pm 13.6^{* *}$ & $35.4 \pm 12.6^{* *}$ \\
\hline $2 \mathrm{~h}$ & $0.553 \pm 0.009$ & $0.18 \pm 0.010^{* *}$ & $0.39 \pm 0.023 * *$ & $76.1 \pm 2.85^{*}$ & $69.8 \pm 6.6^{* *}$ & $44.4 \pm 10.8^{* *}$ & $39.5 \pm 11.5^{* *}$ \\
\hline $2.5 \mathrm{~h}$ & $0.556 \pm 0.010^{*}$ & $0.18 \pm 0.013^{* *}$ & $0.38 \pm 0.020^{* *}$ & $76.0 \pm 3.02 *$ & $68.8 \pm 6.4 * *$ & $45.5 \pm 17.4 * *$ & $41.0 \pm 13.7 * *$ \\
\hline $3 \mathrm{~h}$ & $0.555 \pm 0.021$ & $0.20 \pm 0.011$ & $0.39 \pm 0.025^{* *}$ & $77.3 \pm 3.25^{*}$ & $68.6 \pm 6.3 * *$ & $43.9 \pm 18.6^{* *}$ & $39.7 \pm 14.8^{* *}$ \\
\hline $3.5 \mathrm{~h}$ & $0.557 \pm 0.020$ & $0.18 \pm 0.015^{*}$ & $0.38 \pm 0.022 *$ & $76.3 \pm 2.23 * *$ & $68.0 \pm 6.4^{* *}$ & $42.0 \pm 17.2 *$ & $38.2 \pm 13.8^{* *}$ \\
\hline $4 \mathrm{~h}$ & $0.554 \pm 0.009$ & $0.19 \pm 0.015^{*}$ & $0.41 \pm 0.021 * *$ & $74.0 \pm 2.96$ & $69.3 \pm 6.9 * *$ & $45.7 \pm 17.3^{* *}$ & $39.6 \pm 13.0^{* *}$ \\
\hline
\end{tabular}

fatigue scale ratings generally increased; as a mean value, fatigue scale ratings rose from 3.6 to 7.39. These results indicate that after 4 hours of computer operation, subjects' subjective fatigue increased noticeably.

According to the visual fatigue symptoms after experiment, results indicate that subjects commonly experienced eye discomfort after the 4-hour experiment.

Figure 3 shows the normalized and averaged pulse wave. Each curve represents 30 subjects' average pulse wave in each period. After the first 30 minutes of the experiment, mental fatigue accumulated; the tidal wave became more evident and higher, the height of the dominant wave declined and the docrotie wave trough height increased. Figure 4 shows the normalized and averaged pulse wave before the experiment and at the 4 hour mark. It shows that after the experiment, the pulse wave was smoother than before; thus, the quality of the pulse wave became worse.

In addition, Figure 3 also shows that the variation of pulse waves after 2.5 hours was very low. The waveform became more stable with little change after fatigue accumulated to a certain degree.

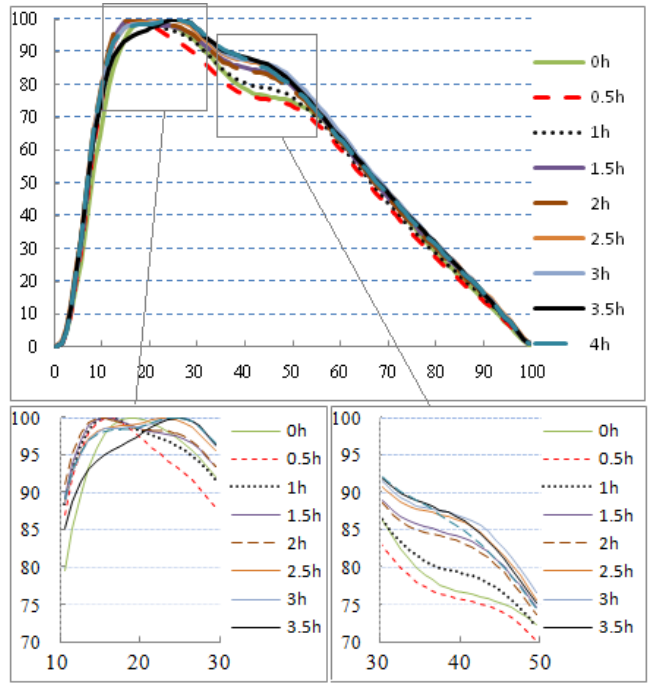

Fig. 3. The pulse wave, which has been normalized and averaged.

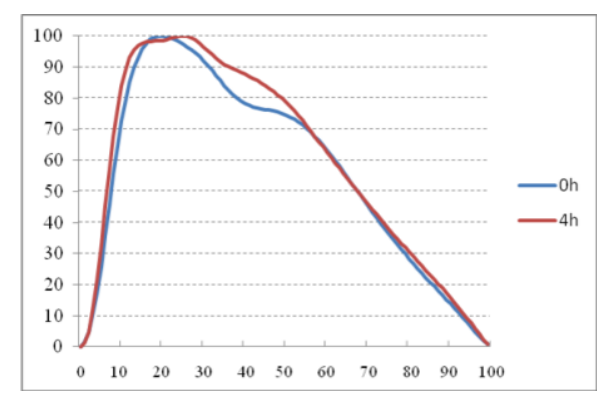

Fig. 4. The pulse wave before the experiment and at 4 hours, normalized and averaged.

Table 1 lists mean values of different time periods during the experiment. T1 indicates the time 
when the dominant wave appeared, T2 indicates the time when the dicrotic wave appeared, D2 indicates the dicrotic wave's strength/height, HR was calculated by counting the peak point of each wave, RMSSD and SDNN indicate pulse rate variation parameters.

Table 1 shows the value, the result of T-Test compared to the starting value, the mean value, and the standard deviation. The heart rate was in a downtrend, while RMSSD and SDNN were in an uptrend. It also shows the changing trends of HR, RMSSD, and SDNN; the HR at the forth hour rose reversely to previous trends. The authors of this experiment believe that was caused by the experimental control. $\mathrm{T} 1$ and $\mathrm{T} 2$ were in a downward trend throughout the experiment $(\mathrm{P}<0.01)$. Their most significant change was in the first hour of the experiment. In the first 30 minutes, the valley height declined; in the following time, it achieved higher height.

After calculation, 30 groups of subjects with P1, P2, P3, P4, P5, P6 and TP were obtained.

From Table 2, it can be seen that the TP of $0-15 \mathrm{~Hz}$ had significant differences $(\mathrm{P}<0.01)$. TP significantly increased; afterward, it became more stable. P1 and P2 remained stable with no significant differences. P3, P4, P5, and P6 displayed obvious differences; the changing trend was the same with TP significantly increasing. The TP was mainly affected by $3-15 \mathrm{~Hz}$.

\section{Discussion and conclusion}

With the popularization of video display terminals, the number of VDT users will likely rise, resulting in increased cases of VDT-caused health issues. This study focused on the VDT-related work fatigue problems. The experiment used pulse wave data to evaluate VDT fatigue. There were changes in the waveform chart, the main wave occurrence time, and the dicrotic wave valley horizontal and vertical coordinates. The tidal wave appeared in the finger volume pulse wave; the main wave and the dicrotic wave valley appeared early, the dicrotic wave valley rose, the overall pulse wave tended to be smooth after the experiment, and the subjects exhibited increased fatigue. In the power spectrum, P3, P4, P5, P6, and TP increased with significant differences $(\mathrm{P}<0.01)$. Therefore pulse wave parameters hold the potential to be an effective means of measuring VDT-caused fatigue.

Table 2

The mean values of TP, P1, P2, P3, P4, P5, and P6

\begin{tabular}{llllllll}
\hline Time/Parameters & $\mathrm{TP}$ & $\mathrm{P} 1$ & $\mathrm{P} 2$ & $\mathrm{P} 3$ & $\mathrm{P} 4$ & $\mathrm{P} 5$ & $\mathrm{P} 6$ \\
\hline 0 & $95.9 \pm 5.3$ & $62.9 \pm 4.5$ & $55.1 \pm 3.3$ & $49.8 \pm 2.6$ & $44.3 \pm 3.3$ & $41.7 \pm 3.3$ & $38.9 \pm 4.0$ \\
$0.5 \mathrm{~h}$ & $97.1 \pm 4.4$ & $62.5 \pm 4.4$ & $55.2 \pm 2.9$ & $50.5 \pm 3.0$ & $45.7 \pm 3.7$ & $42.9 \pm 4.2$ & $41.0 \pm 3.9^{*}$ \\
$1 \mathrm{~h}$ & $99.9 \pm 6.0^{* *}$ & $63.7 \pm 3.7$ & $56.0 \pm 3.3$ & $51.9 \pm 3.6^{* *}$ & $47.3 \pm 3.8^{* *}$ & $44.5 \pm 4.8^{*}$ & $42.5 \pm 4.8^{* *}$ \\
$1.5 \mathrm{~h}$ & $100.2 \pm 3.9^{* *}$ & $63.2 \pm 4.0$ & $55.6 \pm 2.7$ & $51.6 \pm 2.8^{*}$ & $47.0 \pm 3.4^{*}$ & $44.9 \pm 3.5^{* *}$ & $42.9 \pm 3.7^{* *}$ \\
$2 \mathrm{~h}$ & $100.7 \pm 5.5^{* *}$ & $63.7 \pm 3.8$ & $56.2 \pm 2.9$ & $51.9 \pm 3.0^{*}$ & $47.9 \pm 4.4^{* *}$ & $45.4 \pm 4.8^{* *}$ & $43.7 \pm 4.8^{* *}$ \\
$2.5 \mathrm{~h}$ & $100.5 \pm 4.9^{* *}$ & $62.6 \pm 3.3$ & $55.0 \pm 3.5$ & $51.6 \pm 2.9^{*}$ & $47.5 \pm 3.9^{* *}$ & $44.9 \pm 3.7^{* *}$ & $43.3 \pm 4.1^{* *}$ \\
$3 \mathrm{~h}$ & $100.1 \pm 4.9^{* *}$ & $63.2 \pm 4.5$ & $56.3 \pm 3.9$ & $52.3 \pm 4.2^{*}$ & $48.7 \pm 3.5^{* *}$ & $46.3 \pm 3.9^{* *}$ & $43.8 \pm 3.9^{* *}$ \\
$3.5 \mathrm{~h}$ & $100.1 \pm 4.8^{* *}$ & $63.4 \pm 2.9$ & $55.7 \pm 3.0$ & $51.6 \pm 2.9$ & $47.6 \pm 2.9^{* *}$ & $45.1 \pm 3.7^{* *}$ & $43.3 \pm 3.9^{* *}$ \\
$4 \mathrm{~h}$ & $100.2 \pm 4.3^{* *}$ & $62.5 \pm 3.1$ & $55.0 \pm 3.0$ & $50.9 \pm 3.6$ & $47.7 \pm 3.4^{* *}$ & $44.7 \pm 4.0^{* *}$ & $43.2 \pm 4.0^{* *}$ \\
\hline \multicolumn{2}{l}{ Note: ${ }^{*} \mathrm{P}<0.05,{ }^{* *} \mathrm{P}<0.01}$. & & & & & &
\end{tabular}

\section{References}


[1] S.S. Wu, L.H. He and J.Y. Li, Visual display terminal use increases the prevalence and risk of work-related musculoskeletal disorders among Chinese office workers: A cross-sectional study, Journal of Occupational Health 54 (2012), 34-43.

[2] P. Pillastrini, R. Mugnai and L. Bertozzi, Effectiveness of an ergonomic intervention on work-related posture and low back pain in video display terminal operators: A 3 year cross-over trial, Applied Ergonomics 41 (2010), 436-443.

[3] P. Janwantanakul, P. Pensri and V. Jiamjarasrangsri, Prevalence of self-reported musculoskeletal symptoms among office workers, Occupational Medicine 58 (2008), 436-438.

[4] J. Lapointe, C.E. Dionne and C. Brisson, Interaction between postural risk factors and job strain on self-reported musculoskeletal symptoms among users of video display units: A three-year prospective study, Scandinavian Journal of Work Environment \& Health 35 (2009), 134-144.

[5] A. Mazloum, M. Kumashiro and H. Izumi, Quantitative overload: A source of stress in data-entry VDT work induced by time pressure and work difficulty, Industrial Health 46 (2008), 269-280.

[6] C.X. Chen, K. Li and Q.Y. Wu, EEG-based detection and evaluation of fatigue caused by watching 3DTV, Displays 34 (2013), 81-88.

[7] F. Laurent, M. Valderrama and M. Besserve, Multimodal information improves the rapid detection of mental fatigue, Biomedical Signal Processing and Control 8 (2013), 400-408.

[8] D.P. Bernstein, Pressure pulse contour-derived stroke volume and cardiac output in the morbidly obese patient, Obesity Surgery 18 (2008), 1015-1021.

[9] H.M. Seong, J.S. Lee and T.M. Shin, The analysis of mental stress using time-frequency distribution of heart rate variability signal, 26th Annual International Conference of the IEEE on Engineering in Medicine and Biology Society 1 (2004), 283-285.

[10] L. Salahuddin, J. Cho and M.G. Jeong, Ultra short term analysis of heart rate variability for monitoring mental stress in mobile settings, 29th Annual International Conference of the IEEE on Engineering in Medicine and Biology Society, 2007, Lyon, pp. 4656-4659.

[11] J.S. Wong, W.A. Lu and K.T. Wu, A comparative study of pulse rate variability and heart rate variability in healthy subjects, Journal of Clinical Monitoring and Computing 26 (2012), 107-114.

[12] A.H. Khandoker, C.K. Karmakar and M. Palaniswami, Comparison of pulse rate variability with heart rate variability during obstructive sleep apnea, Medical Engineering \& Physics 33 (2011), 204-209.

[13] E. Gil, M. Orini and R. Bailón, Photoplethysmography pulse rate variability as a surrogate measurement of heart rate variability during non-stationary conditions, Physiological Measurement 31 (2010), 1271.

[14] J.A.J. Heathers, Smartphone-enabled pulse rate variability: An alternative methodology for the collection of heart rate variability in psychophysiological research, International Journal of Psychophysiology 89 (2013), 297-304. 